(DAPI) stained for cell nuclei was performed. Co-localization of IL-33 and DAPI in CD34 positive cells demonstrates IL-33 staining in the nucleus of glomerular endothelial cell of lupus kidney. Expression of intracellular but not surface ST2 was increased in plasmacytoid dendritic cells/pDC (CD16-CD14-CD85k+CD123 + ) of lupus patients when compared with healthy controls. Incubation of HUVECs with IL-33 and/or IL-12 increased the production of IL-6, but IL-4, IL-5, IL-6, IL-13 and TNF- $\alpha$ was not produced.
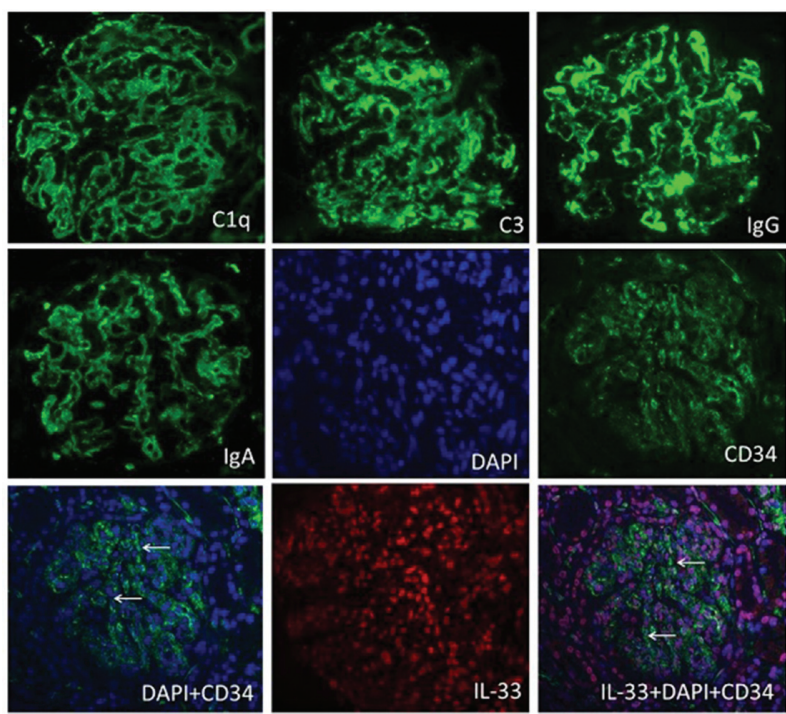

Conclusions: As a result of external stimuli or infection, renal glomerular endothelial cells undergo cellular death and release the "Alarmin", IL-33, to alert the lupus immune system. Released IL-33 interact with their target cells, pDC via their specific receptor ST2 to subsequently induce innate and adaptive responses, activate inflammatory pathways in the pathogenesis of lupus nephritis.

Acknowledgements: This work was supported by National Science Foundation of China (81300585), Science and Technology Planning Project of Guangdong Province (2014A020212322) and Natural Science Foundation of Guangdong Province (2015A030313477).

Disclosure of Interest: None declared

DOI: 10.1136/annrheumdis-2018-eular.6064

\section{SAT0044 LIN28A IS OVEREXPRESSED IN OSTEOARTHRITIS AND IS ESSENTIAL FOR THE STABILITY AND HIGH LEVEL EXPRESSION OF IL-6 AND COX-2 IN HUMAN CHONDROCYTES}

I. Ahmad, M.Y. Ansari, M.N. Khan, T.M. Haqqi. Anatomy and Neurobiology, North East Ohio Medical University, Rootstown, USA

Background: Osteoarthritis (OA), the most common type of joint disease, is characterised by progressive and irreversible degradation of articular cartilage. Dysregulated gene expression has also been linked to disease pathogenesis. Lin28A is an evolutionarily conserved RNA binding protein and known to play a critical role in development, metabolism and tumorigenesis. However, the role of Lin28A in osteoarthritis is not yet explored.

Objectives: To study the role of Lin28A in the regulation of genes associated with OA pathogenesis in human chondrocytes.

Methods: Primary human chondrocytes were isolated from the undamaged portion of the knee OA cartilage by enzymatic digestion and were cultured in DMEM/ F12\% and $10 \%$ FCS. Total RNA from cartilage explants or chondrocytes was prepared using Trizol and was made DNA-free by on-column digestion method. mRNA expression levels of Lin28A, Lin28B, MMP-13, IL-6, COX2 and iNOS were quantified by TaqMan assays. Protein expression was analysed by immuno-blotting using validated antibodies. Nucleofection was used for the siRNA mediated depletion or plasmid mediated overexpression of Lin28A gene was used to study its role in chondrocyte function under pathological conditions. RNA immunoprecipitation (RIP) with anti-Lin28A antibody was performed to identify the interacting mRNA partners. Stability of the mRNAs was determined by Actinomycin-D chase experiments.

Results: Human OA cartilage samples $(n=6)$ analysed were found to express Lin28A but not Lin28B transcripts. Lin28A mRNA expression was significantly high in the damaged $\mathrm{OA}$ cartilage compared to the smooth cartilage from the same patient $(n=3, p<0.05)$. Stimulation with $I L-1 \beta$ induced the high levels of Lin28A mRNA and protein expression in OA chondrocytes in a time dependent as well as dose dependent manner. siRNA mediated depletion of Lin28A expression in $\mathrm{OA}$ chondrocytes inhibited the IL-1 $\beta$-induced expression of MMP-13, IL-6, COX2 and iNOS mRNAs. Importantly, the overexpression of Lin28A induced the expression of MMP-13, IL-6, COX-2 and iNOS mRNA and protein in OA chondrocytes. Immunoblotting analysis showed that the Lin28A depleted OA chondro cytes treated with IL-1 3 also produced significantly low levels of IL-6, MMP-13 and COX-2 protein compared to chondrocytes transfected with scrambled siRNAs $(n=3, p<0.05)$. RIP analyses in IL-1 $\beta$ treated OA chondrocytes with anti-Lin28A antibody revealed that the MMP-13, IL-6 and COX2 mRNAs were pulled down with anti-Lin28A and were highly enriched when compared with the MRNA population pulled down by isotype control antibody. siRNA mediated depletion of Lin28A expression resulted in decreased half-life of IL-6 and COX-2 mRNAs, while the overexpression of Lin28A had the opposite effect in IL-1 $\beta$ stimulated OA chondrocytes. This indicated that Lin28A contributes towards the stability of IL-6 and COX-2 mRNAs.

Conclusions: Our data for the first time demonstrate that Lin28A plays a key role in OA pathogenesis by stabilising the expression of catabolic gene transcripts in human chondrocytes under pathological conditions. These data revealed a previously unidentified role of Lin28A in chondrocytes and identify it as a potential therapeutic target for the treatment of $O A$.

Acknowledgements: Supported by NIH grants RO1 AT-007373, RO1 AR067056 and funds from the Northeast Ohio Medical University to TMH.

Disclosure of Interest: I. Ahmad: None declared, M. Ansari: None declared, M. Khan: None declared, T. Haqqi Grant/research support from: USPHS Grants DOI: 10.1136/annrheumdis-2018-eular.6011

\section{SAT0045 DNA METHYLATION OF SOCS3 AS A POSSIBLE MECHANISM FOR PERSISTENT URATE INDUCED INFLAMMATION}

V. Klück ${ }^{1}$, T. Crisan ${ }^{2}$, M. Cleophas ${ }^{1}$, S. Keating ${ }^{1}$, B. Novakovic ${ }^{3}$, N. Dalbeth ${ }^{4}$, L. Stamp ${ }^{5}$, A. Phipps-Green ${ }^{6}$, M. Black ${ }^{6}$, D. Macartney-Coxson ${ }^{7}$, T. Merriman ${ }^{6}$, L. Joosten'. ' ${ }^{1}$ Experimental Internal Medicine, Radboudumc, Nijmegen, Netherlands; ${ }^{2}$ Department of Medical Genetics, Iuliu Hatieganu University of Medicine and Pharmacy, Cluj-Napoca, Romania; ${ }^{3}$ Department of Molecular Biology, Radboud University, Nijmegen, Netherlands; ${ }^{4}$ Department of Medicine, University of Auckland, Auckland; ${ }^{5}$ Department of Medicine, University of Otago, Christchurch; ${ }^{6}$ Department of Biochemistry, University of Otago, Dunedin; Environmental and Scientific Research, Wellington, New Zealand

Background: Hyperuricemia is a metabolic condition associated with cardiovascular diseases. ${ }^{1}$ However, mechanisms for a causal relation have not been fully elucidated yet. Previously, we showed that monocytes primed with urate show a shift in the balance of cytokine production: increased proinflammatory cytokines and decreased levels of IL-1 receptor antagonist. ${ }^{2}$

Objectives: In this study we investigate if these changes to urate exposure are persistent and whether changes in DNA methylation serve as a molecular substrate for these effects of hyperuricemia.

Methods: DNA methylation was assessed in whole blood of 80 individuals of Maori ancestry with varying serumurate levels. Human PBMCs and monocytes from Dutch healthy volunteers were isolated and pretreated for 24 hour with urate. Cells were either directly stimulated with LPS or LPS/MSU or subjected to increasing resting days before restimulation. Cytokine levels were determined in supernatants by ELISA. SOCS3 mRNA levels were determined by qPCR after 24 hour urate priming. Phosphorylation of STAT3 was assessed after stimulation by flow cytometry with intracellular staining for PSTAT3.

Results: Human PBMCs primed with urate demonstrated increased IL-1 $\beta$ and IL6 responses and decreased IL-1Ra production compared to controls. Although IL$1 \beta$ production was diminished after increasing resting days, persistent effects were observed for the reduction of IL-1Ra and induction of IL-6. To investigate whether these persistent changes were mediated by epigenetic changes, differences in DNA methylation between normouricemic and hyperuricemic individuals were assessed. SOCS3 gene was higher methylated at 3 neighbouring SOCS3 intragenic positions in hyperuricemic individuals. In vitro, SOCS3 mRNA levels were significantly increased in monocytes after 24 hour urate treatment. Moreover, urate dose-dependently suppressed the phosphorylation of STAT3 after stimulation.

Conclusions: In this study we demonstrated that urate has persistent proinflammatory effects on human monocytes. Higher SOCS3 DNA methylation is observed in hyperuricemic individuals. In vitro, urate priming leads to increased levels of SOCS3 mRNA and consequently suppression of STAT3 phosphorylation. Interestingly, STAT3 inhibition has been reported to mediate IL-1Ra downregulation. ${ }^{3}$ Therefore, we hypothesize urate induced inflammation is at least partly mediated by changes in methylation of the SOCS3 gene. However, further validation of this pathway is needed to elucidate possible targets for therapy. 


\section{REFERENCES:}

[1] Soltani Z, et al. Potential role of urate in metabolic syndrome, hypertension, kidney injury, and cardiovascular diseases: is it time for reappraisal? Ann Rheum Dis 2016;75(4):755-62.

[2] Crisan TO, et al. Soluble urate primes TLR-induced proinflammatory cytokine production by human primary cells via inhibition of IL-1Ra. Ann Rheum Dis 2016;75(4):755-62.

[3] Tamassia N, et al. Uncovering an IL-10-dependent NF-kappaB recruitment to the IL-1ra promoter that is impaired in STAT3 functionally defective patients. Faseb j 2010;24(5):1365-75.

Disclosure of Interest: None declared

DOI: 10.1136/annrheumdis-2018-eular.6281

\section{SAT0046 TRANSCRIPT-PROTEIN ASSOCIATIONS IN EARLY RA PATIENTS ACHIEVING SUSTAINED DRUG-FREE REMISSION AFTER TREATMENT WITH TOCILIZUMAB}

X.M. Teitsma ${ }^{1}$, J.W. Jacobs ${ }^{1}$, A.N. Concepcion ${ }^{1}$, A. Pethö-Schramm ${ }^{2}$, M.E. Borm ${ }^{3}$ J.M. van Laar', J.W. Bijlsma' ${ }^{1}$, F.P. Lafeber ${ }^{1} .{ }^{1}$ Rheumatology and Clinical Immunology, UMC Utrecht, Utrecht, Netherlands; ${ }^{2}$ F Hoffmann-La Roche, Basel, Switzerland; ${ }^{3}$ Roche Nederland BV, Woerden, Netherlands

Background: In disease modifying anti-rheumatic drug (DMARD)-naïve early rheumatoid arthritis (RA) patients, we previously identified networks of differentially co-expressed genes associated with achieving sustained drug-free remission (sDFR). ${ }^{1} \mathrm{~A}$ better understanding of processes involved with translation of mRNA into proteins might be used to develop personalised treatment for early RA patients.

Objectives: To identify inflammatory proteins associated with achieving sDFR by performing multi-analyte profiling in pre-treatment serum and subsequently to study significantly enriched biological pathways and compare these with the pathways previously found in the transcriptomic analyses.

Methods: In this exploratory study, baseline serum was analysed of 24 patients ( $n=13$ achieved sDFR, $n=11$ controls) treated-to-target with tocilizumab (TCZ) therapy in the U-Act-Early trial. TCZ (intravenously, $8 \mathrm{mg} / \mathrm{kg}$ ) was given every 4 weeks; if no remission, hydroxychloroquine (HCQ) was added and if subsequently remission was still not achieved, HCQ was replaced by (oral) methotrexate. Thereafter, if the target, remission, still was not achieved, patients switched to standard of care (e.g. tumour necrosis factor inhibitor). Provided remission persisted, therapy was tapered and subsequently discontinued. SDFR was reached when patients remained $>3$ months in remission while being drug-free until the end of the study period; those not able to discontinue therapy were selected as controls. Luminex multi-analyte profiling (xMAP) ${ }^{\oplus}$ was used to measure 85 inflammatory proteins; partial least square discriminant analyses (PLS-DA) was applied to identify relevant proteins associated with achieving SDFR.

Results: No significant differences in clinical baseline characteristics were found between those achieving sDFR vs controls $(p>0.05)$. PLS-DA identified 14 proteins of which $6 / 14(n / N)$ were associated with a decreased chance of achieving sDFR. The protein considered most important (i.e. highest variable on importance (VIP) score) was chemokine (C-C motif) ligand 20 (CCL20, VIP 1.49). In total, 88 significantly enriched Gene Ontology (GO) terms were identified analysing the proteins; 5 terms, of which "positive regulation of leukocyte migration" $\left(p \leq 3.41^{\mathrm{E}-02}\right)$ and "leukocyte chemotaxis" $\left(p \leq 3.49^{\mathrm{E}-03}\right)$ were found in both proteomic and transcriptomic analyses. Significant transcript-protein correlations are shown in figure 1; the TIMP metallopeptidase inhibitor 1 (TIMP-1) protein showed the highest number $(n=12)$ of correlations.

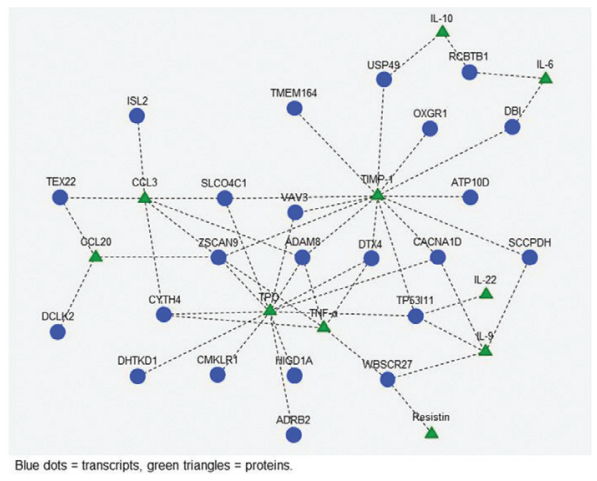

Abstract SAT0046 - Figure 1. Network visualisation of significant transcript-protein correlations.
Blue dots=transcripts, green triangles=proteins.

Conclusions: Several relevant proteins were found associated with achieving sDFR after treatment with TCZ. When performing pathway analyses, several enriched pathways within the protein biomarkers were also previously identified in the transcriptomic analyses, providing further insight into gene expression and translation of inflammatory proteins in early RA patients.

\section{REFERENCE:}

[1] Teitsma XM, Jacobs JWH, Mokry M, et al. Identification of differential coexpressed gene networks in early rheumatoid arthritis achieving sustained drug-free remission after treatment with a tocilizumab-based or methotrexate-based strategy. Arthritis Res Ther 2017;19:170.

Disclosure of Interest: X. Teitsma: None declared, J. Jacobs Grant/research support from: The department of the author (JWGJ) who included patients in the U-Act-Early trial received reimbursements from Roche Nederland BV., A. Concepcion: None declared, A. Pethö-Schramm Employee of: AP-S is an employee of $\mathrm{F}$ Hoffmann-La Roche, M. Borm Employee of: MEAB is an employee of Roche Nederland BV, J. van Laar Grant/research support from: JMvL received fees from Arthrogeen, MSD, Pfizer, Eli Lilly, and BMS and research grants from Astra Zeneca, Roche-Genentech., J. Bijlsma Grant/research support from: JWJB reported grants and fees from Roche, AbbVie, Bristol-Myers Squibb, Merck Sharp and Dohme, Pfizer, and UCB., F. Lafeber Grant/research support from: FPJL reports grants from Roche.

DOI: 10.1136/annrheumdis-2018-eular.3467

\section{SAT0047 PTEN REGULATION ALLEVIATES THE ALCOHOL- INDUCED OSTEOPENIA IN RAT VIA AKT/GSK-3B/B- CATENIN PATHWAY IN BMSCS}

\section{Y. Chen, Y. Gao, C. Zhang. Orthopedic, Shanghai Sixth People hospital,} Shanghai, China

Background: Alcohol is regarded as a leading risk factor of osteopenia. Our pre-

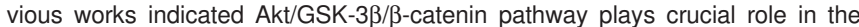
ethanol-induced anti-osteogenic effect in bone mesenchymal stem cells (BMSCs). It was acknowledged that PI3K/Akt is negatively regulated by the phosphatase and tensin homologue (PTEN) phosphatase. PTEN expression was reported to be upregualted in ethanol-administrated animals.

Objectives: In this study, we explored the molecular mechanisms underlying

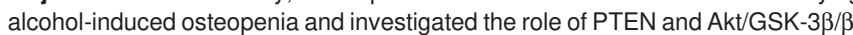
catenin axis in this pathological process.

Methods: In vitro, Western blotting, separation of nucleus and cytosolic extracts confocal scanning, RT-PCR were used to investigate the inhibition of ethanol on Akt/GSK3 $\beta / \beta$-catenin signalling pathway via upregulation of PTEN in hBMSCs. In vivo, micro-computerised tomography, hematoxylin and eosin $(\mathrm{H}$ and $\mathrm{E})$ staining, Van Gieson staining, Masson's trichrome and fluorochrome labelling were employed to reveal that PTEN inhibition provided protective effects against ethanol on bone tissue.

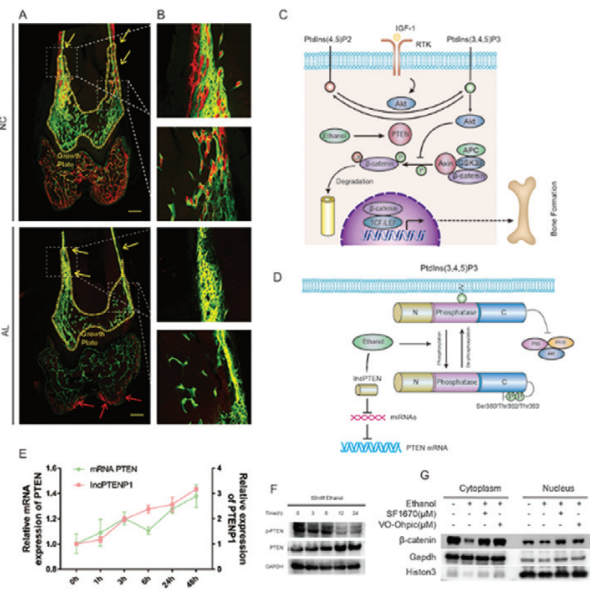

Results: We found that ethanol increased PTEN expression both in BMSCs and in bone tissue of ethanol-administrated rats. PTEN upregulation impaired the recruitment of Akt to the plasma membrane, and suppressed Akt phosphorylation at Ser473, there by inhibiting the Akt/GSK3// $\beta$-catenin signalling pathway in BMSCs and inhibited the expression of osteogenic genes $C O L 1$ and $O C N$ both in vitro and in vivo. To counteract the inhibitory effect of ethanol, two selective PTEN inhibitors were introduced. The result of micro-computerised tomography, 